\title{
A Novel Model for Boron Diffusion in SiGe Strained Layers Based on a Kinetics Driven Ge-B Pairing Mechanism
}

\author{
D. Villanueva, P. Moens \\ Alcatel Microelectronics, Westerring 15, B-9700 Oudenaarde, Belgium \\ davy.villanueva@mie.alcatel.be \\ K. Rajendran, W. Schoenmaker \\ IMEC, Kapeldreef 75, B-3001 Leuven, Belgium
}

\begin{abstract}
A new temperature dependant model for the Ge-B pairing mechanism has been established and implemented in a process simulator. The combination of both lattice strain effects and $\mathrm{GeB}$ clustering has been successfully applied to various anneals. The match with experimental SIMS profiles is excellent.
\end{abstract}

\section{Introduction}

Controlling the B outdiffusion in SiGe strained layers is a major concern since it rules the electrical properties of high frequency bipolar transistors. Studies have been carried out describing how Ge lowers the B diffusivity ${ }^{(1-3)}$. It appears that the effect of strain in the lattice and a sort of clustering reaction between $\mathrm{Ge}$ and $\mathrm{B}$ are the most relevant mechanisms to investigate. In this paper, results from simulations featuring both phenomena are presented. The novelty is that the clustering reaction is no longer rendered by an additional term to Fick's law ${ }^{(1)}$ but is now treated as a chemical reaction containing the models for kinetics and thermodynamics. Appropriate SIMS profiles after various relevant RTP and furnace anneals have allowed to precise the reaction equation for the Ge-B clustering.

\section{Experimental Data}

The calibration of the presented model is based on SIMS profiles analysis. Wafers have been processed with a B box shaped profile $(8 \mathrm{e} 18 / \mathrm{cm} 3)$ partly included in a $\mathrm{Ge}$ graded profile (up to $7.5 \mathrm{e} 21 / \mathrm{cm} 3$ ). In order to emphasise chemical reactions, the samples have been submitted to different thermal budgets, ranging from 1000 to $1100^{\circ} \mathrm{C}$. Another set of SIMS profiles based on a three B spikes profile in a Ge box profile and submitted to typical furnace anneals, has been used to check the validity of the presented model. 


\section{Diffusion model \& Clustering Reaction}

The diffusion model is largely based on the one presented by Cowern et al. ${ }^{(3)}$, including the effect of lattice strain on the B diffusivity. It also features the dependency of the intrinsic carrier concentration on $\mathrm{Ge}$ as presented by Rajendran et al. ${ }^{(1)}$.

The main focus of this work is the mathematical treatment of the pairing mechanism between $\mathrm{Ge}$ and $\mathrm{B}$. When including large $\mathrm{Ge}$ atoms in the $\mathrm{Si}$, the lattice is compressed in the growth plane and expanded in the growth direction, which leads to a strained structure. Due to the smaller size of B atoms compared to $\mathrm{Si}$ atoms, a local tensile strain exists around the $\mathrm{B}$ atoms. On the contrary $\mathrm{Ge}$ atoms will generate local compressive strain points. In order to realise some stress energy, B and Ge atoms will attract each other and thus create GeB clusters. This mechanism can be modelled as follows:

- The first solution is to include the trapping mechanism as an additional term to

Fick's law ${ }^{(1)}$ :

$$
\frac{\partial C_{B}}{\partial t}=-\nabla J_{B}-\frac{C_{B}}{\tau_{G e B}}
$$

In this way, very good results can be achieved for temperatures in the range between 800 and $1050^{\circ} \mathrm{C}$, but it fails for higher temperatures, as shown in Figure 1.

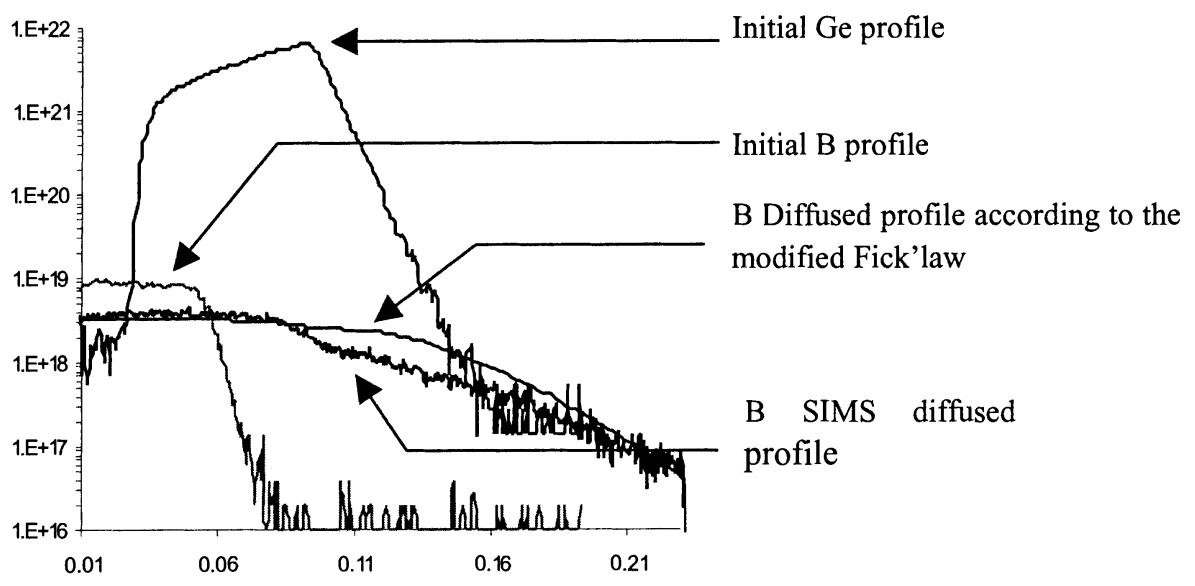

Fig.1. Failure of the modified Fick's law model at $1100^{\circ} \mathrm{C}$ during $40 \mathrm{sec}$

- The second option is to go for a chemical reaction between $\mathrm{Ge}$ and $\mathrm{B}$ as defined by thermodynamics and kinetics. This solution suggests that, since the clustering mechanism is seen as a chemical reaction, it should be thermally enhanced and consequently the study will be mainly focussed on the samples with the highest temperatures. In general, the reaction term will be defined as follows: 


$$
R=\left[a \exp \left(\frac{-E}{k T}\right) \cdot \sum_{m, n} a_{m, n}[G e]^{m}[B]^{n}\right] \cdot K_{0}
$$

Basically, the resultant term consists of a kinetic part, which is weighted by the thermodynamic factor $\mathrm{K}_{0}$.

As a starting point, it will be assumed that the reaction is not limited by thermodynamics, and hence the main focus is on kinetic considerations. Such assumptions may be justified by the fact that the thermodynamic equilibrium is not very likely to be reached within a few seconds, i.e. during most RTP steps.

From a large number of simulations and from the comparison of the simulated data with the experimental SIMS profiles, it was found that at least two terms are required in order to reproduce the observed features. The most simple reaction rate equation is:

$$
\text { Rate }=\alpha[G e] \bullet[B]+\beta[G e]^{m \geq 2}
$$

The first term is responsible for the $\mathrm{GeB}$ cluster formation at medium Ge concentrations, whereas the second term is required to simulate the cluster formation at higher $\mathrm{Ge}$ concentrations. In this way, the enhanced B pile-up at high Ge concentrations is well reproduced. The parameters of equation [3] have been determined by fitting various experimental SIMS profiles.

\section{Results \& Discussions}

The simulations were performed by implementing equation [3] in the process simulator DIOS included in the ISE TCAD tools package ${ }^{(4)}$. Figure 2 shows the simulated $\mathrm{B}$ profile after a $40 \mathrm{sec}$ anneal at $1100^{\circ} \mathrm{C}$.

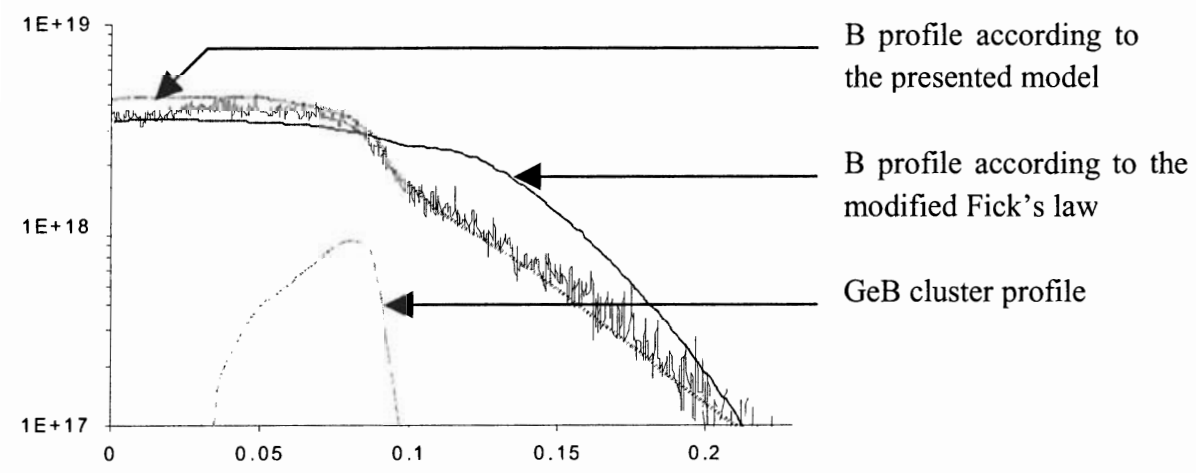

Fig.2. B profiles after $40 \sec @ 1100^{\circ} \mathrm{C}$

A perfect match with the experimental profile is obtained only with the new model. It follows that up to $30 \%$ of the $\mathrm{B}$ atoms are trapped into GeB clusters, resulting in a significantly retarded diffusion for high Ge doping level. The GeB cluster profile 
exhibits two peaks: one at $0.5 \mu \mathrm{m}$ depth and another at $0.8 \mu \mathrm{m}$ depth, due to the first and second terms in the reaction equation respectively. The presented model has also been applied to the "three spikes" B profile of reference ${ }^{(1)}$ annealed at $900^{\circ} \mathrm{C}$ during $12 \mathrm{~min}$. For the $12 \mathrm{~min}$ anneal, the simulated profile follows very closely the experimental one, whereas only including strain cannot mimic the B peak, as shown in Figure 3.

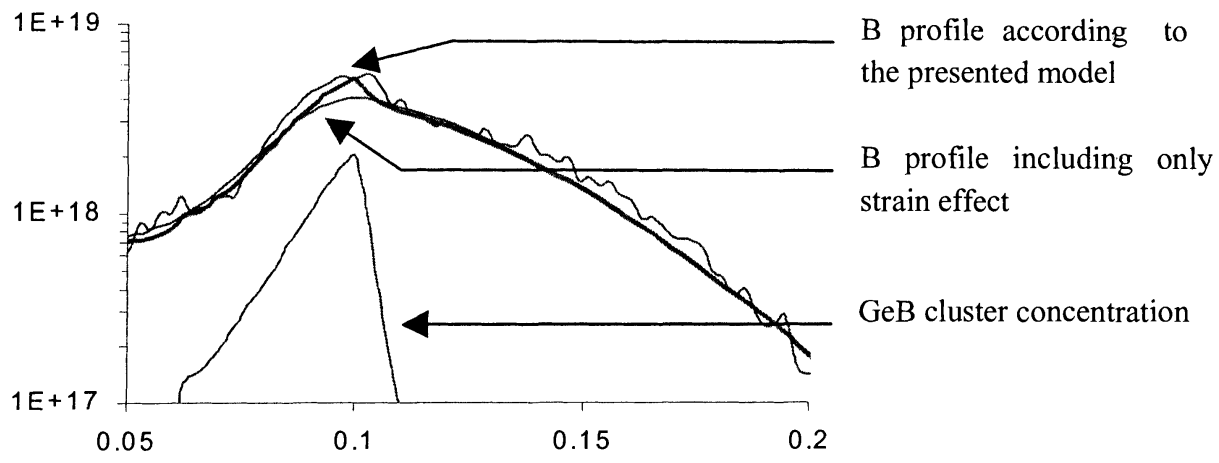

Fig.3. B profiles from the three spikes profile after a $12 \mathrm{~min}$ anneal at $900^{\circ} \mathrm{C}$

Even at such temperature, it is interesting to notice that up to $40 \%$ of the B concentration may be trapped by Ge. The efficiency of the presented model for this anneal largely overshoots the limits defined by the thermodynamic assumptions, and thus extends its applicable range to most of the usual thermal budgets for processing.

\section{Conclusion}

It has been shown that modelling the $\mathrm{B}$ retarded diffusion requires the use of a temperature dependent $\mathrm{GeB}$ clustering model. Therefore the chemical approach has proven to be the most adequate and flexible solution over a wide temperature range.

\section{Acknowledgements}

The authors wish to thank H. Ziad and P. Chevalier for their contribution. Part of this work was performed in the frame of the MEDEA T555 project.

\section{References}

[1] K. Rajendran et al., Simulation of B Diffusion in SiGe layers. SISPAD2000, pp. 206-209

[2] R.F. Lever et al., Boron diffusion across silicon-silicon germanium boundaries. J. Appl. Phys., vol.83, pp.1988-1994, 1998.

[3] N. Cowern et al., Diffusion in strained (SiGe), Phys. Rev. Lett., vol.72, pp. 2585, 1994.

[4] DIOS, process simulator, Ver 6.1, ISE AG, Zurich, Switzerland. 